\title{
A New Approach to Discover Students Learning Styles in Adaptive Educational Systems
}

\author{
Fabiano A. Dorça \\ Faculdade de Computação \\ Universidade Federal de \\ Uberlândia - Campus Sta. \\ Mônica - Uberlândia - MG \\ fabiano@,facom.ufu.br
}

\author{
Luciano V. Lima \\ Faculdade de Engenharia \\ Elétrica - Universidade \\ Federal de Uberlândia - \\ Campus Sta. Mônica - \\ Uberlândia - MG \\ vieira@ufu.br
}

\author{
Márcia A. Fernandes \\ Faculdade de Computação \\ Universidade Federal de \\ Uberlândia - Campus Sta. \\ Mônica - Uberlândia - \\ MG \\ marcia@ufu.br
}

\author{
Carlos R. Lopes \\ Faculdade de Computação \\ Universidade Federal de \\ Uberlândia - Campus Sta. \\ Mônica - Uberlândia - \\ MG \\ crlopes@,ufu.br
}

\begin{abstract}
Personalization according to specific requirements of an individual student is one of the most important features in adaptive educational systems. Considering learning and how to improve a student's performance, these systems must know the way in which an individual student learns best. In this context, the current work outlines a new approach to automatically and dynamically discover students learning styles, considering its non-deterministic and non-stationary aspects, and taking into account that learning styles may change during the learning process in an unexpected and unpredictable way. Our approach is mainly based in genetic algorithms and reinforcement learning, and it has been tested through computer simulation of students. Promising results have been obtained through experiments. Some of them are presented in this paper. Keywords-learning styles, student modelling, e-learning, adaptive educational systems.
\end{abstract}

Keywords: automatic and dynamic student modelling; learning styles; adaptive and intelligent educational systems; probabilistic student modelling. 


\section{Introduction}

Most of the distance education systems don't take into account the individual student's characteristics. As a consequence, the same learning objects, pedagogical strategies and learning resources are commonly used for everybody. However, adaptivity [4] has been introduced in these systems during the last years in order to provide individual learning experience [18]. In this way, the main student's characteristics considered in the personalization of a course are learning goals [9], cognitive level [9], interests [4], stereotypes [41] and learning styles (LS) [13]. Considering LS in adaptive educational systems (AES) is motivated by educational and psychological theories, which argue that learners have different ways in which they prefer to learn [18].

LS and their effects on learning processes are carefully exposed by Coffield [11]. Their related instructional strategies have been massively studied in the new learning space introduced by the Internet, where many researchers point out that linking LS to appropriate learning resources is an important stimulus for the learning process. Some researches reveal that students performances improves if the learning environment supports their specific LS. On the other hand, learners whose LS are not supported by the learning environment may have more difficulties during the learning process $[24,22,28,2,23,13]$.

In order to provide adaptivity, the students characteristics have to be known first. However, the traditional approaches for detection of LS in AES are inefficient. Price [34] analyses the uncertainty aspect related to ILS (Index of Learning Styles Questionnaire) by identifying inconsistencies between its results and students behaviours. Besides Price, Roberts [35] analyses this kind of instrument and the problems related to it. Therefore, many approaches for automatic detection of LS have been proposed. However, in general they present problems which make them either inefficient or difficult to implement, implant and use, as pointed out in section 2 .

In this context, we propose a new approach to discover students LS, which automatically detects and precisely adjusts students LS based on the non-deterministic and non-stationary aspects of LS, that may change, or evolve, during the learning process in an unexpected and unpredictable way [18]. Our approach is based on the Felder and Silverman Learning Styles Model (FSLSM) [13]. According to Graf and Kinshuk [18, 19], the FSLSM uses the concept of dimensions, and therefore describes LS in much detail.

A very important characteristic of FSLSM to our work is that it uses scales to classify students instead of defined types. In this way, the strength of each LS is finely measured [13]. Therefore, our approach aims to gradually fine tune LS stored in SM along the learning process in order to efficiently optimise the SM. Another important aspect of FSLSM is that it considers preferences as tendencies and therefore, it takes into account that the student may act differently in specific situations, in a non-deterministic way, as pointed out by Kinshuk et al [28] and by Graf et al [18].

Therefore, we consider students preferences as probabilities in the four-dimensional FSLSM model, as depicted in section 3. As a result, our approach gradually, constantly and stochastically modifies students LS using a set of rules which infer how the SM should be updated at a specific moment. The SM is updated through a reinforcement learning [37] based approach, as pedagogical actions are selected through a genetic algorithm [17]. As a consequence, the student model (SM) effectively converges towards the real student's LS, as shown through experiments in section 4 . Finally, section 5 presents conclusions and future work.

\section{Related Work}

A diversity of approaches for automatic detection of LS have been proposed, such as [21, 20, 8]. In general, these traditional approaches use deterministic inference systems for detecting students LS through predefined behavioural patterns. These systems infer LS based on students actions. A problem with these systems is the difficulty to develop rules that are able to infer LS effectively through students actions and to treat students behaviour as evidences and not as a possibilities.

More complex approaches can be seen in $[27,16,6$, $5,43,42,7]$. These approaches use learning machine techniques, such as Bayesian and neural networks. A problem with these approaches is their high complex implementations and high computation cost, which is a serious concern when there is a high number of simultaneous students using the system. Besides, in general, these approaches are highly coupled either to the system they were designed for or to the whole process aimed at selecting the suitable learning resources according to the student's specific characteristics, making them harder to re-use in other systems. In some of these approaches, once acquired, the student's LS remains the same throughout the entire learning process [8].

Another well-known problem with learning machine approaches is the complication generated by concept drift and concept shift [8]. They occur either because the acquired LS information needs to be adjusted or because students simply change their preferences. In this scenario, 
adaptive decision models that are able to better fit students preferences are desirable.

In this context, we believe that our approach brings advantages due to some specific characteristics:

- considering that not only LS but many factors have an influence on students performances (knowledge and behavior), making it harder to infer students LS based only on fixed behavioural pattern rules. Some of these factors are pointed out in [24, 22, 28, 2, 23];

- considering that students LS can change over time in an unpredictable way [18] and that these changes may be associated with other factors, such as knowledge domain, as analysed by Jones [26];

- considering that, as pointed out in [34], it's impossible to know about the correctness of results obtained from self-assessment questionnaires, where a deterministic process should take a very long time in order detect inconsistencies or may never detect it;

- eliminating the necessity of discovering students behavioural patterns, considering that it's hard to obtain such patterns. They may have inconsistencies and they make the student modelling process deterministic;

- using reinforcement learning instead of standard supervised learning, which differs mainly because in reinforcement learning correct input/output pairs are never presented, nor sub optimal actions explicitly corrected, and further, there is a focus on on-line performance, which is a great feature when detecting students LS;

- being uncoupled from any Learning Management System (LMS), and independent of students actions in a specific system, as it occurs in traditional approaches, as [16, 21];

- the consideration that LS have a dynamic nature, and they may change when the knowledge domain changes [27] or naturally evolve over time [30].

The next section presents important aspects of our approach.

\section{Implementation Issues}

In this section we present our approach in detail, which uses information from students performances for updating student model (SM) frequently while they use the system for learning. The SM, which may have incon- sistencies related to LS, gradually and stochastically evolves toward the real student's LS.

In this way, the information about students LS is dynamically and constantly revised and corrected while learning difficulties are detected, leading to an accurate and fine-tuned SM, which contributes to more accurate adaptivity.

Student's LS are stored in SM as values in the interval $[0,1]$ representing a student's probability of preference for a specific LS in a FSLSM dimension. Therefore, the student's LS are stored as probability distributions. If a selfassessment questionnaire is used for initialization of probabilistic LS (LSp), as ILS [14], the SM can be booted from the data obtained by the questionnaire, considering the proportion of responses scored for each LS inside a dimension. If any self-assessment questionnaire is used, LSp is initialized with 0.50 (undefined preference). Using this approach, a student's LS are represented according to Definition 3.1.

Definition 3.1: Learning Styles (LS)

$L S=\left\{\left(\operatorname{Pr}_{A}, \operatorname{Pr}_{R}\right),\left(\operatorname{Pr}_{S}, \operatorname{Pr}_{I}\right),\left(\operatorname{Pr}_{V i}, \operatorname{Pr}_{V e}\right)\right.$, $\left(P r_{S e q}, P r_{G}\right) \mid P r_{A}+P r_{R}=1, P r_{S}+P r_{I}=1, P r_{V i}+$ $\left.\operatorname{Pr}_{V e}=1, \operatorname{Pr}_{S e q}+\operatorname{Pr}_{G}=1\right\}$

where:

$\operatorname{Pr}_{A}$ is the probability of the student's preference for the Active LS;

$\operatorname{Pr}_{R}$ is the probability of the student's preference for the Reflective LS;

$\operatorname{Pr}_{S}$ is the probability of the student's preference for the Sensitive LS;

$\operatorname{Pr}_{I}$ is the probability of the student's preference for the Intuitive LS;

$P r_{V i}$ is the probability of the student's preference for the Visual LS;

$P r_{V e}$ is the probability of the student's preference for the Verbal LS;

$P r_{S e q}$ is the probability of the student's preference for the Sequential LS;

$\operatorname{Pr}_{G}$ is the probability of the student's preference for the Global LS.

In addition, our approach is based on probabilistic learning styles combinations [15]. A learning style combination is a 4-tuple composed by one LS from each FSLSM dimension, as stated by Definition 3.2.

Definition 3.2: Learning Styles Combination (LSC) $L S C=(a, b, c, d) \mid a \in D 1, b \in D 2, c \in D 3, d \in D 4$ where:

$D 1=\{\operatorname{Active}(A), \operatorname{Reflective}(R)\}$

$D 2=\{\operatorname{Sensitive}(S)$, Intuitive $(I)\}$

$D 3=\{\operatorname{Visual}(\operatorname{Vi}), \operatorname{Verbal}(V e)\}$

$D 4=\{\operatorname{Sequential}(\operatorname{Seq}), \operatorname{Global}(G)\}$ 
Therefore, there are 16 possible learning styles combinations, as stated by Definition 3.3.

Definition 3.3: Learning Styles Combinations (LSCs) $\mathrm{LSCs}=\{(\mathrm{A}, \mathrm{Vi}, \mathrm{S}, \mathrm{Seq}), \quad(\mathrm{A}, \mathrm{Vi}, \mathrm{S}, \mathrm{G}), \quad(\mathrm{R}, \mathrm{Vi}, \mathrm{S}, \mathrm{Seq})$, (R,Vi,S,G), (A,Ve,S,Seq), (A,Ve,S,G), (R,Ve,S,Seq), (R,Ve,S,G),(A,Vi,I,Seq), (A,Vi,I,G), (R,Vi,I,Seq), (R,Vi,I,G), (A,Ve,I,Seq), (A,Ve,I,G), (R,Ve,I,Seq), $(\mathrm{R}, \mathrm{Ve}, \mathrm{I}, \mathrm{G})\}$.

We propose that during each different learning session the student should interact with a set of learning objects (LO) [25] which satisfies a specific LSC, stochastically selected according to the student's LS preferences stored in the SM. Which means that, in our approach, a LSC is a specific combination of four random variables [33]. Therefore, in our approach, the student's LS describes the probability of random variables $\mathrm{a}, \mathrm{b}, \mathrm{c}$ and $\mathrm{d}$, considering Definition 3.2.

For example, considering a student's probable LS, $\mathrm{LSp}=\{(0.35,0.65), \quad(0.17,0.83), \quad(0.89,0.11)$, $(0.84,0.16)\}$, we can consider that this student probably is (R)eflective, (I)ntuitive, (Vi)sual and (Seq)uential. Taking into consideration that LS are probabilities, a LSC has a probability to be selected during a learning session which is equal to the student's probabilistic preference $(\mathrm{P})$ for the LSC.

Table 1 - LSC Probability Distribution

\begin{tabular}{|c|c|}
\hline LSC & Probabilities \\
\hline $\mathrm{P}(\mathrm{A}, \mathrm{S}, \mathrm{Vi}, \mathrm{Seq})$ & $0,35 \times 0,17 \times 0,89 \times 0,84=0,045$ \\
\hline $\mathrm{P}(\mathrm{A}, \mathrm{S}, \mathrm{Vi}, \mathrm{G})$ & $0,35 \times 0,17 \times 0,89 \times 0,16=0,008$ \\
\hline $\mathrm{P}(\mathrm{R}, \mathrm{S}, \mathrm{Vi}, \mathrm{Seq})$ & $0,65 \times 0,17 \times 0,89 \times 0,84=0,083$ \\
\hline $\mathrm{P}(\mathrm{R}, \mathrm{S}, \mathrm{Vi}, \mathrm{G})$ & $0,65 \times 0,17 \times 0,89 \times 0,16=0,016$ \\
\hline $\mathrm{P}(\mathrm{A}, \mathrm{S}, \mathrm{Ve}, \mathrm{Seq})$ & $0,35 \times 0,17 \times 0,11 \times 0,84=0,005$ \\
\hline $\mathrm{P}(\mathrm{A}, \mathrm{S}, \mathrm{Ve}, \mathrm{G})$ & $0,35 \times 0,17 \times 0,11 \times 0,16=0,002$ \\
\hline $\mathrm{P}(\mathrm{R}, \mathrm{S}, \mathrm{Ve}, \mathrm{Seq})$ & $0,65 \times 0,17 \times 0,11 \times 0,84=0,010$ \\
\hline $\mathrm{P}(\mathrm{R}, \mathrm{S}, \mathrm{Ve}, \mathrm{G})$ & $0,65 \times 0,17 \times 0,11 \times 0,16=0,003$ \\
\hline $\mathrm{P}(\mathrm{A}, \mathrm{I}, \mathrm{Vi}, \mathrm{Seq})$ & $0,35 \times 0,83 \times 0,89 \times 0,84=0,217$ \\
\hline $\mathrm{P}(\mathrm{A}, \mathrm{I}, \mathrm{Vi}, \mathrm{G})$ & $0,35 \times 0,83 \times 0,89 \times 0,16=0,043$ \\
\hline $\mathrm{P}(\mathrm{R}, \mathrm{I}, \mathrm{Vi}, \mathrm{Seq})$ & $0,65 \times 0,83 \times 0,89 \times 0,84=0,403$ \\
\hline $\mathrm{P}(\mathrm{R}, \mathrm{I}, \mathrm{Vi}, \mathrm{G})$ & $0,65 \times 0,83 \times 0,89 \times 0,16=0,076$ \\
\hline $\mathrm{P}(\mathrm{A}, \mathrm{I}, \mathrm{Ve}, \mathrm{Seq})$ & $0,35 \times 0,83 \times 0,11 \times 0,84=0,026$ \\
\hline $\mathrm{P}(\mathrm{A}, \mathrm{I}, \mathrm{Ve}, \mathrm{G})$ & $0,35 \times 0,83 \times 0,11 \times 0,16=0,005$ \\
\hline $\mathrm{P}(\mathrm{R}, \mathrm{I}, \mathrm{Ve}, \mathrm{Seq})$ & $0,65 \times 0,83 \times 0,11 \times 0,84=0,049$ \\
\hline $\mathrm{P}(\mathrm{R}, \mathrm{I}, \mathrm{Ve}, \mathrm{G})$ & $0,65 \times 0,83 \times 0,11 \times 0,16=0,009$ \\
\hline Sum of probabilities & 1.000 \\
\hline
\end{tabular}

Therefore, considering the student's LS above, we have $\mathrm{P}(\mathrm{R}, \mathrm{I}, \mathrm{Vi}, \mathrm{Seq})=0.83 \times 0.89 \times 0.65 \times 0.84=$
$0.403(40.3 \%)$. The sum of the rest of the probabilistic preferences is equal to $0.597(59.7 \%)$. The sum of all $\mathrm{P}$ (LSC) in LSCs is equal to $1.000(100 \%)$. Therefore, according to the LS above, (R,I,Vi,Seq) is the preferred one. But, we have to consider that the student's LS may have inconsistencies, and in this case the system doesn't know the real student's preferences.

In this context, the most advantage of our approach is to stochastically consider all LSCs according to the student's probable LS, that may be wrong or may change over time, as pointed out in 2 . As shown in section 4 , this characteristic allows to effectively discover and fine-tune the real student's LS (denoted by LSr).

Taking into consideration that LS are probabilities, a specific LSC may be probably selected during a learning session according to the probability distribution shown in Table 1, considering the LSp given above.

The selection of a LSC is done by a stochastic selection method [17]. There are a variety of stochastic selection methods, for example Roulette Wheel Selection, Stochastic Universal Sampling, Simple Tournament and Stochastic Tournament [17].

In this approach we use the Roulette Wheel Selection, due to it's adequacy to our approach. In this method, individual candidates have a fitness that measures how adapted they are to the environment [17], which gives them a proportional slice in the roulette. The individual's probability of selection is given by the proportion between its fitness and the entire population's fitness, as shown in (1). The sum of probabilities of all the individuals must equal 1 .

$$
\operatorname{Pr}_{i}=\frac{\text { fitness }_{i}}{\sum_{x=1}^{n} \text { fitness }_{x}}
$$

So, we calculate the accumulated probability for each individual, as shown in (2).

$q_{0}=0 ; q_{1}=q_{0}+P r_{1} ; \ldots ; q_{n}=q_{n-1}+P r_{n}=1$

Finally, we generate a random number in the interval $[0,1]$ representing the roulette spin. If the generated number is in the interval [q0, q1], the individual 1 is selected. If the number is in the interval [q1, q2], the individual 2 is selected, and so on, as shown in (3).

$$
q_{i-1}<\operatorname{Pr}_{i} \leq q_{i}
$$


In our approach, we consider the probability of selecting a specific LSC as its probability of preference by the student, as shown in Table 1. Applying (2) we have the following accumulated probabilities related to LSCs:

$$
\begin{aligned}
& q_{0}=0 \\
& q_{1}=0+\operatorname{Pr}_{(A, S, V i, S e q)}=0.045 \\
& q_{2}=0.045+\operatorname{Pr}_{(A, S, V i, G)}=0.053 \\
& q_{3}=0.053+\operatorname{Pr}_{(R, S, V i, S e q)}=0.136 \\
& q_{4}=0.136+\operatorname{Pr}_{(R, S, V i, G)}=0.152 \\
& q_{5}=0.152+\operatorname{Pr}_{(A, S, V e, S e q)}=0.157 \\
& q_{6}=0.157+\operatorname{Pr}_{(A, S, V e, G)}=0.159 \\
& q_{7}=0.159+\operatorname{Pr}_{(R, S, V e, S e q)}=0.169 \\
& q_{8}=0.169+\operatorname{Pr}_{(R, S, V e, G)}=0.172 \\
& q_{9}=0.172+\operatorname{Pr}_{(A, I, V i, S e q)}=0.389 \\
& q_{10}=0.389+\operatorname{Pr}_{(A, I, V i, G)}=0.432 \\
& q_{11}=0.432+\operatorname{Pr}_{(R, I, V i, S e q)}=0.835 \\
& q_{12}=0.835+\operatorname{Pr}_{(R, I, V i, G)}=0.911 \\
& q_{13}=0.911+\operatorname{Pr}_{(A, I, V e, S e q)}=0.937 \\
& q_{14}=0.937+\operatorname{Pr}_{(A, I, V e, G)}=0.942 \\
& q_{15}=0.942+\operatorname{Pr}_{(R, I, V e, S e q)}=0.991 \\
& q_{16}=0.991+\operatorname{Pr}_{(R, I, V e, G)}=1.000
\end{aligned}
$$

Applying (3) we have:

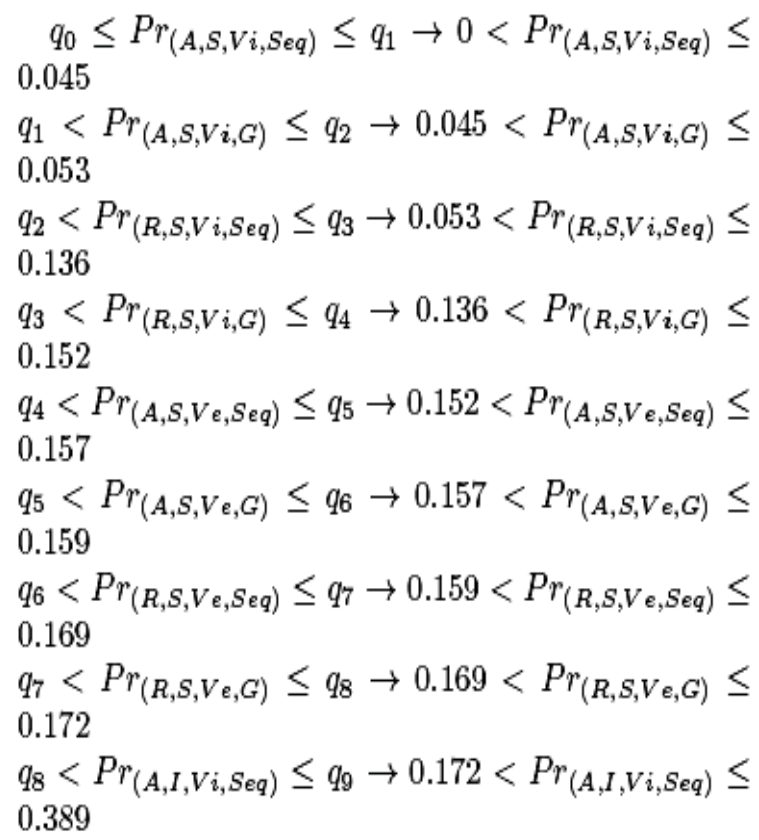

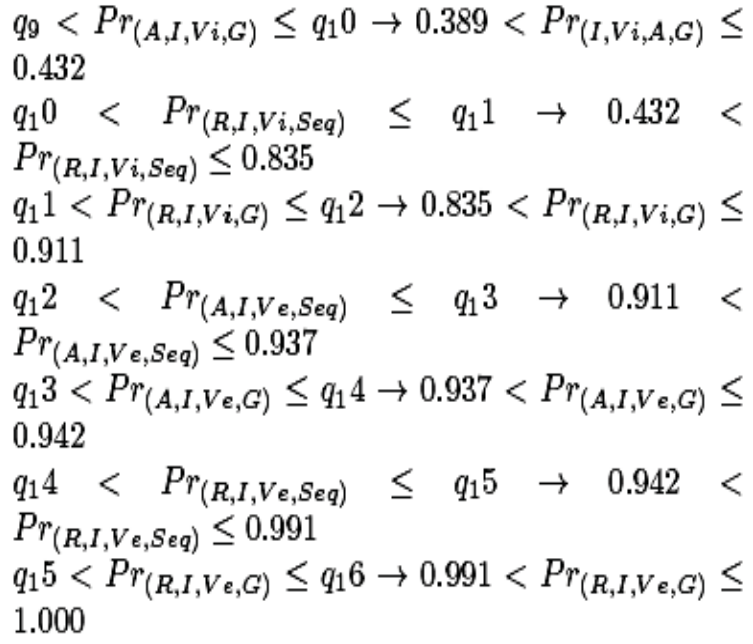

Once each LSC have been assigned a fitness value, they can be chosen with probability given by their relative fitness. A binary representation of LSCs is used, where preferences A, S, Vi, Seq are represented by 0 and preferences $\mathrm{R}, \mathrm{I}, \mathrm{Ve}, \mathrm{G}$ are represented by 1 . Therefore, we have the following $\mathrm{LSCs}=\{(0,0,0,0),(0,0,0,1),(1,0,0,0)$, $(1,0,0,1),(0,1,0,0),(0,1,0,1),(1,1,0,0),(1,1,0,1),(0,0,1,0)$, $(0,0,1,1), \quad(1,0,1,0), \quad(1,0,1,1), \quad(0,1,1,0), \quad(0,1,1,1)$, $(1,1,1,0),(1,1,1,1)\}$. If population size is greater than 16 , the first 16 individuals are copied from LSCs and the rest of the individuals are randomly generated.

After each learning session, we apply recombination and mutation operators, based on genetic algorithms [10]. The recombination operator recombines LSCs in order to (probably) produce more fitted individuals. During recombination, the parents are stochastically selected through Roulette Wheel Selection method. The role of parent selection is to distinguish among individuals based on their quality, in order to allow the better individuals to become parents of the next generation. We are using here the single-point crossover [10]. This crossover operation is not necessarily performed on all strings in population. Instead, it is applied with a probability $P x$ when the pairs are chosen for breeding.

The mutation operator is then applied to the new LSC with a probability $\mathrm{Pm}$ (mutation rate). Mutation causes the individual genetic representation to be changed according to some probabilistic rule. In the binary string representation, mutation will cause a single bit to flip, 0 to 1 or 1 to 0 . The bit to be flipped is randomly chosen. For example, the individual (R,I,Vi,Seq) may be changed through mutation to (A,I,Vi,Seq).

It's important to consider that LSp stored in SM are constantly updated. In order to decide how LS must be updated, it is taken into account the LSC selected during a learning session. When the student shows a learning problem during a learning session, the student's preferences in 
SM that accords to the selected LSC are decremented, considering a probable inconsistency in these preferences. The student's preferences in SM that discords to the selected LSC are incremented, making them stronger, considering that the learning difficulties appeared because they were not present in the selected LSC. This heuristic guides the process of automatic student modelling and is implemented by the following production rules (where A and $\mathrm{B}$ represents a $\mathrm{LS}$ in a specific dimension):

$$
\begin{gathered}
(P F M<m) A N D(L S C[i]=A) \rightarrow \\
L S_{p}[i]_{A}:=L S_{p}[i]_{A}-\alpha \times R, \\
L S_{p}[i]_{B}:=L S_{p}[i]_{B}+\alpha \times R . \\
(P F M<m) A N D(L S C[i]=B) \rightarrow \\
L S_{p}[i]_{A}:=L S_{p}[i]_{A}+\alpha \times R, \\
L S_{p}[i]_{B}:=L S_{p}[i]_{B}-\alpha \times R .
\end{gathered}
$$

such that:

- LSp[i]A is the probability of preference for the LS "A" stored in the SM, in dimension $\mathrm{i}$, with $\mathrm{i}=$ $1 . .4$.

- $\mathrm{LSp}[\mathrm{i}] \mathrm{B}$ is the probability of preference for the LS "B" stored in the SM, in dimension $\mathrm{i}$, with $\mathrm{i}=$ $1 . .4$.

- LSC [i] is the LS that appears in the LSC, considering the dimension $\mathrm{i}$, with $\mathrm{i}=1 . .4$.

- $0<\alpha<1$ is the learning rate, which indicates how fast the system learns about students LS.

Because of the probabilistic and dynamic factors enclosed on automatic detection of LS, our approach gradually and constantly modifies the SM through reinforcement learning, considering students performances as rewards of pedagogical actions, which are given by LSCs. In this way, LSCs are actions that the system can execute with the intention of teaching content, maximizing the quality of students performances in the learning process.

Therefore, the reinforcement is calculated according to the performance value(PFM) obtained by the student during a learning session, according to (4).

$$
R=\frac{1}{P F M \times D L S}
$$

$$
D L S=\left|S M\left[d_{i}\right]_{A}-S M\left[d_{i}\right]_{B}\right|
$$

The reinforcement is inversely related to performance, since, probably, the lower the performance, the greater the difficulty of learning, which can probably be caused by strong inconsistency in LSp which should be eliminated as soon as possible, requiring greater reinforcement.

By the other side, it is desirable that the greater the distance between LS (DLS) in a dimension, the lower the reinforcement, so that we can avoid abrupt increases on a considerably strong LSp, and allowing a greater increase when DLS value approximates to 0 (undefined preference).

The PFM value is considered in the interval $[0,100]$ and the DLS value is considered in the interval $[0,1]$. A variable Rmax limits the value of $R$, with the intention of preventing too large reinforcements when DLS or PFM tends to 0 .

It's well-known that a variety of factors should be taken into account for calculating student's performance, as pointed out in [12]. It's a complex problem and a lot of approaches have been proposed to solve it. For testing our approach without this complexity, we developed a student simulator that calculates students performances taking into account some aspects related to the impact of LS on student's performance, as depicted in [24, 22, 28, 2, 23].

The main aspect of simulating students performances is that when a student's LS is not satisfied by the selected LSC, it makes learning harder and decreases learning efficiency and performance. The simulator takes into consideration how many student's LS are not satisfied by the selected LSC, and if strong preferences are not supported, which improves difficulty too. A probabilistic model was designed to represent the non-deterministic aspect encapsulated in students performances, due to the large amount of factors that influence it.

Simulation is a widespread and widely used technique for testing educational approaches and may bring advantages, as stated by [1, 38, 40, 39, 3, 29, 31].

The student simulator is not focused on this paper and it will be depicted at another opportunity. The next section presents some experiments and discusses the results.

\section{Testing and Validating the Pro- posed Approach}

The proposed approach has been tested through a set of experiments. Some of them are expounded in this section and their results are discussed. The experiments expounded in this section were executed considering the following parameters:

- population size $=100$, where the first 16 indi- 
viduals are copied from LSCs (see Definition 3.3 ) and the rest of the individuals are randomly generated.

- $\mathrm{Px}=0.2$

- $\mathrm{Pm}=0.1$

Furthermore, we considered that good performances (no learning problem detected) occurs when PFM $\geq 60$, and bad performances (learning problem detected) occurs when $\mathrm{PFM}<60$. The learning rate, $\alpha$, was set to 0.8 , and the limit value for the reinforcement, Rmax, was set to 0.2 .

We considered a set of 30 concepts to be learned by the student. We considered the students initial cognitive level, in all concepts, equivalent to the first level of the Bloom's Taxonomy [36]. Moreover, it was considered as learning objectives the highest level of knowledge in all concepts. The execution of an experiment is completed when the student reaches all learning objectives specified in the SM. The student's cognitive state in a given concept evolves through six levels, arranged in a hierarchy, from least complex to most complex.

Therefore, the simulated learning process in these experiments must have at least 180 learning sessions (or iterations) in order to achieve all learning goals (30 concepts $\times 6$ cognitive levels $=180$ ). The student's cognitive level advances if $P F M \geq m$, and does not advance if PFM $<\mathrm{m}$, where $\mathrm{m}=60$ in these experiments. Therefore, the easier the learning process, fewer iterations are needed in order to reach all learning goals. As the learning process becomes harder, more iterations are necessary to achieve the learning goals.

In order to validate the proposed approach, two variables were observed in the experiments:

- consistency: LSp (student's probable LS) effectively converge to LSr (student's real LS) during the learning process?

- efficiency: LSp converges to LSr in a reasonable time, ie, LSp becomes consistent at the beginning of the learning process?

It is important to notice that without using simulation, it would be difficult to validate this approach. This is because if testing the proposed approach on real learning process, with real students, it would be impossible to know students LSr exactly, due to the reasons pointed out previously, regarding uncertainty and inconsistency of data obtained with traditional LS modelling approaches. Thus, it would be impossible to measure the consistency of the resulting LSp, due to the uncertainty about the LSr.

For each experiment, we set up the student's LSr and the strength of each preference (strong, moderate, weak or balanced). For each experiment, we present graphically the process of updating LSp during the learning process. In each graph, the axis $\mathrm{x}$ shows the number of iterations of the learning process, and the axis y shows the values of LSp (multiplied by 100) throughout the learning process in each dimension of the FSLSM. The main objective was to observe how LSp is gradually updated throughout the learning process in different contexts. Thus, it was possible to clearly visualize the process of automatic detection of students LS.

It is also presented the average performance and average learning problems occurred at intervals of 20 iterations. Therefore, it is possible to see how the average performance increases and the amount of learning problems decreases, as LSp is fixed and becomes consistent with LSr. It was verified that occurrences of learning problems was reduced, as a result of the improvement of students performances, as LSp became consistent with LSr.

Each experiment was repeated 20 times so that we could observe our approach working under different circumstances but under identical conditions. In each experiment we considered different initial assumptions about the student's LS. In addition, we set the real student's LS, used by the student simulator. The student simulator needs to know the LSr and the strength of each one (strong, moderate, weak or balanced). Five experiments and their results are shown in this section.

\subsection{Experiment 1}

First, we considered a student with $\mathrm{LSr}=$ \{reflective (strong), sensitive (strong), visual (moderate), global (weak) \}. The LSp in SM are initially defined as $\mathrm{LSp}=\{(0.70,0.30),(0.35,0.65),(0.60,0.40),(0.45,0.55)\}$

As it can be seen, LS are initially inconsistent and doesn't express all the student's preferences correctly, specifically in dimensions active/reflective and sensitive/ intuitive. Figure 1 presents one execution of this experiment and shows how the student's LS changes along the learning process's iterations. All repetitions in this experiment produced a consistent SM through a different path, due to the non-deterministic aspect of the student modelling process.

As it can be seen in Figure 1, approximately 192 iterations were necessary to finish the learning process. The initially inconsistent dimensions, active/reflective and sensitive/intuitive, were corrected in the very beginning of the learning process, satisfying the evaluation of this approach, considering consistency and efficiency aspects.

The initially consistent dimensions, visual/verbal and 


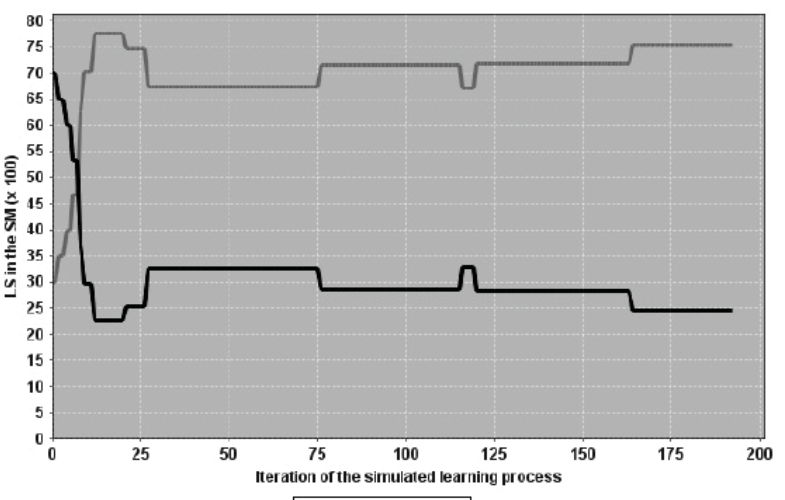

- Active - Reflective

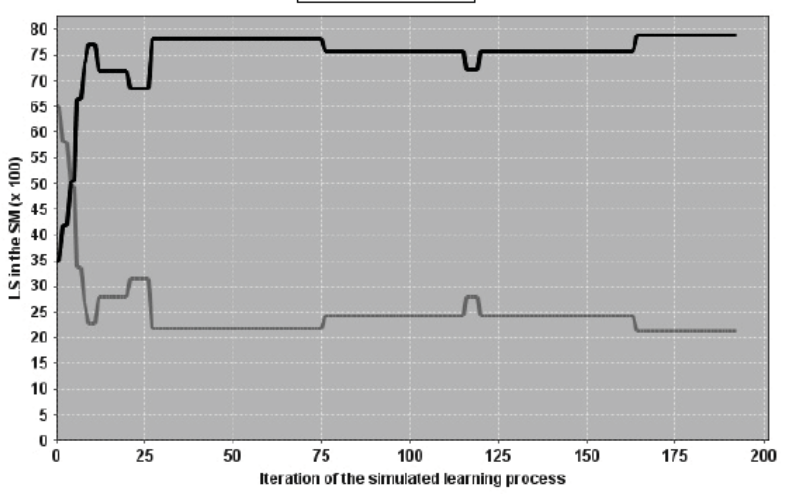

- Sensitive - Intuitive

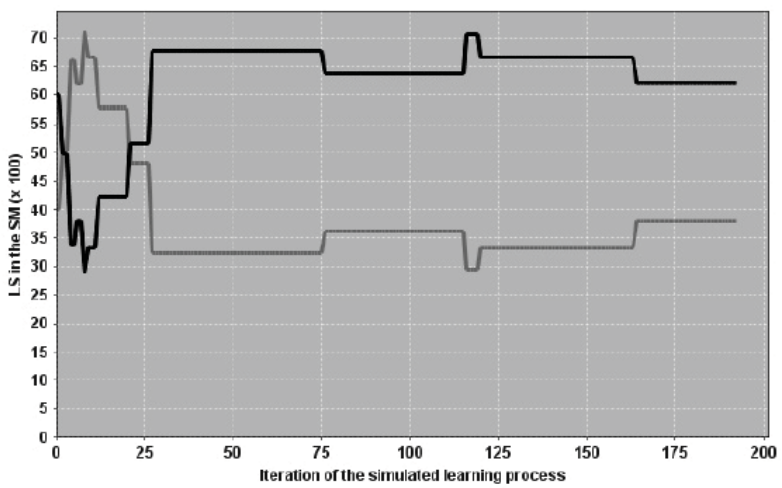

-Visual - Verbal

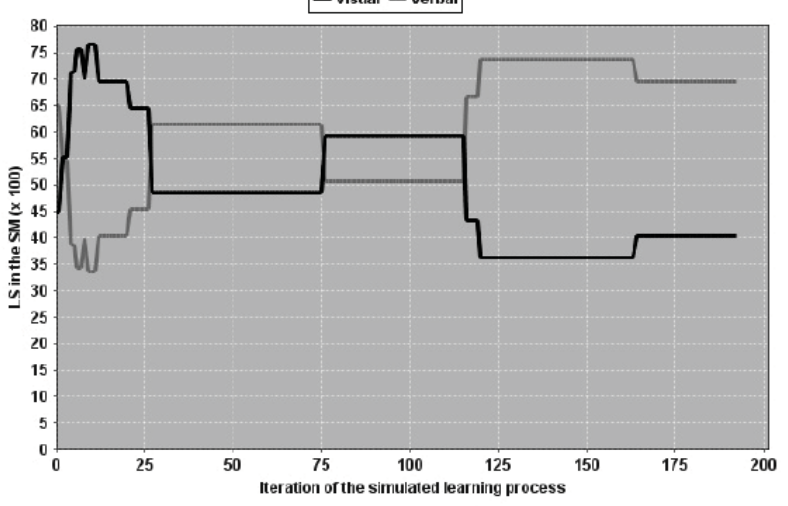

- Sequential - Global

Fig. 1: Results from Experiment 1 sequential/global, were inconsistent midway, but became consistent in the end of the learning process. This occurrence highlights the dynamic and non-deterministic aspects enclosed in our approach, which uses reinforcement learning in order to update LSp in SM.

It is important to mention that reinforcement learning differs from supervised learning techniques in that correct input/output pairs are never presented, nor suboptimal actions explicitly corrected. Rather, there is a focus on on-line performance, which involves finding a balance between exploration (of uncharted actions) and exploitation (of current knowledge) [37].

Figure 2 shows the average performance achieved by the student and the average learning problems in steps of 20 learning sessions. As it can be seen, learning problems were considerably reduced, as dimensions active/reflective and sensitive/intuitive became consistent in SM.

Figure 3 shows the population fitness (sum of the overall individuals fitness). The population fitness was increased throughout the learning process, enabling wellfitted learning process.
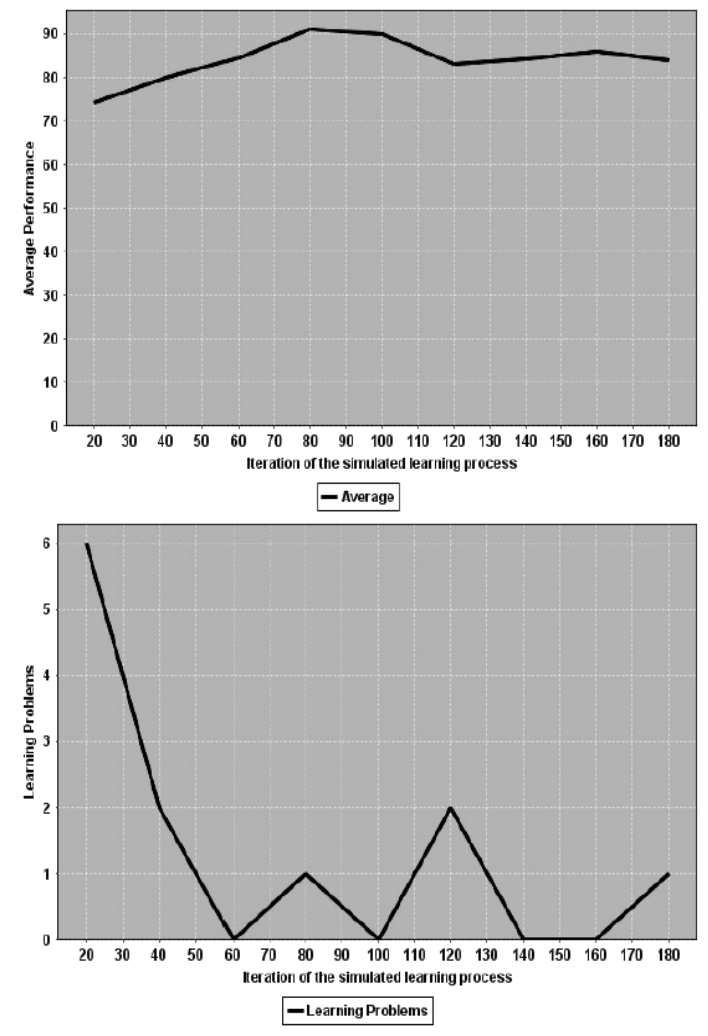

Fig. 2: Average performances and average learning problems in experiment 1 


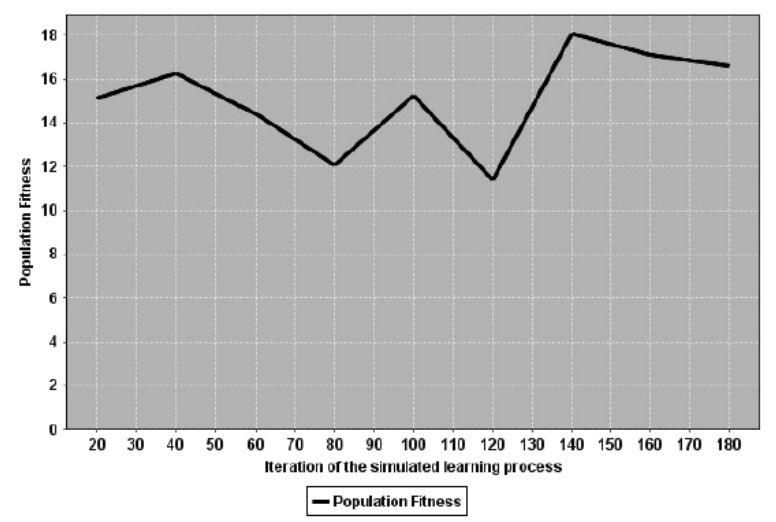

Fig. 3: Population Fitness during experiment 1

\subsection{Experiment 2}

In the following experiment, we consider the case in which there was no initial information available about students LS, which means LSp $=\{(0.50,0.50)$, $(0.50,0.50),(0.50,0.50),(0.50,0.50)\}$. We considered LSr $=\{$ reflective (weak), intuitive (strong), visual(moderate), sequential (weak) $\}$.

Figure 4 presents the results obtained from an execution of this experiment. As it can be seen, in dimensions active/reflective, sensitive/intuitive and visual/verbal, LS were inferred inconsistently in the very beginning of the learning process, but they became consistent and stable soon. Again, this behaviour highlights the dynamic and non-deterministic aspects enclosed in automatic and dynamic detection of students LS.

Figure 5 shows the average performance achieved by the student and the average learning problems in steps of 20 learning sessions. As it can be seen, learning problems were considerably reduced, and average performance was increased, while population fitness (in Figure 6) was increased, allowing a well-fitted adaptivity process.

Figure 6 shows the population fitness (sum of the overall individuals fitness). Particularly in this experiment, the initial population fitness was very low if compared to other experiments, because the SM (used in the calculation of individuals fitness) lacked information about student's LSp. But, the population fitness was quickly increased, as the SM became consistent.

\subsection{Experiment 3}

This experiment presents a case in which we re-use a consistent SM obtained from experiment 2. The goal is to show the behaviour of the learning process when the SM is initially consistent. In this experiment, students initial $\mathrm{LSp}=\{(0.39,0.61),(0.29,0.71),(0.68,0.32),(0.69,0.31)\}$

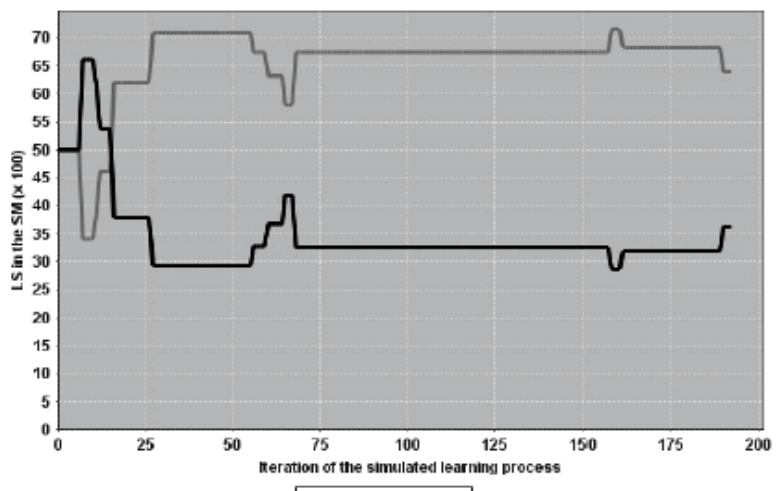

- Active - Reflective

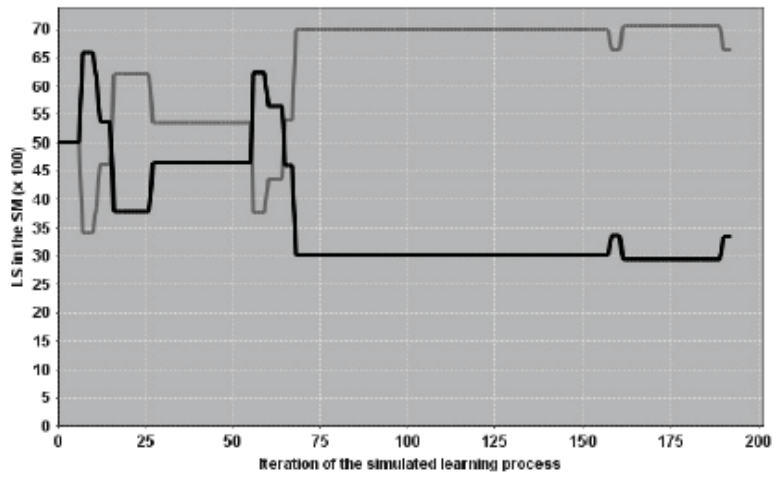

- Sensitive - intuitive
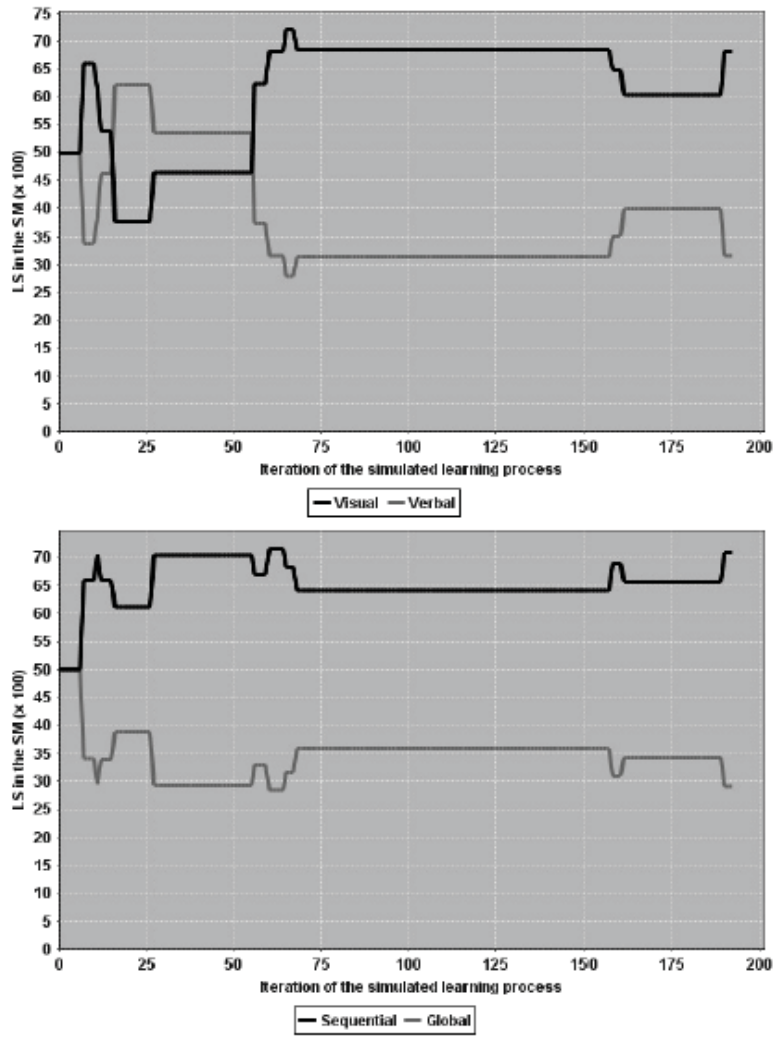

Fig. 4: Results from Experiment 2 
A New Approach to Discover Students Learning Styles in Adaptive Educational systems
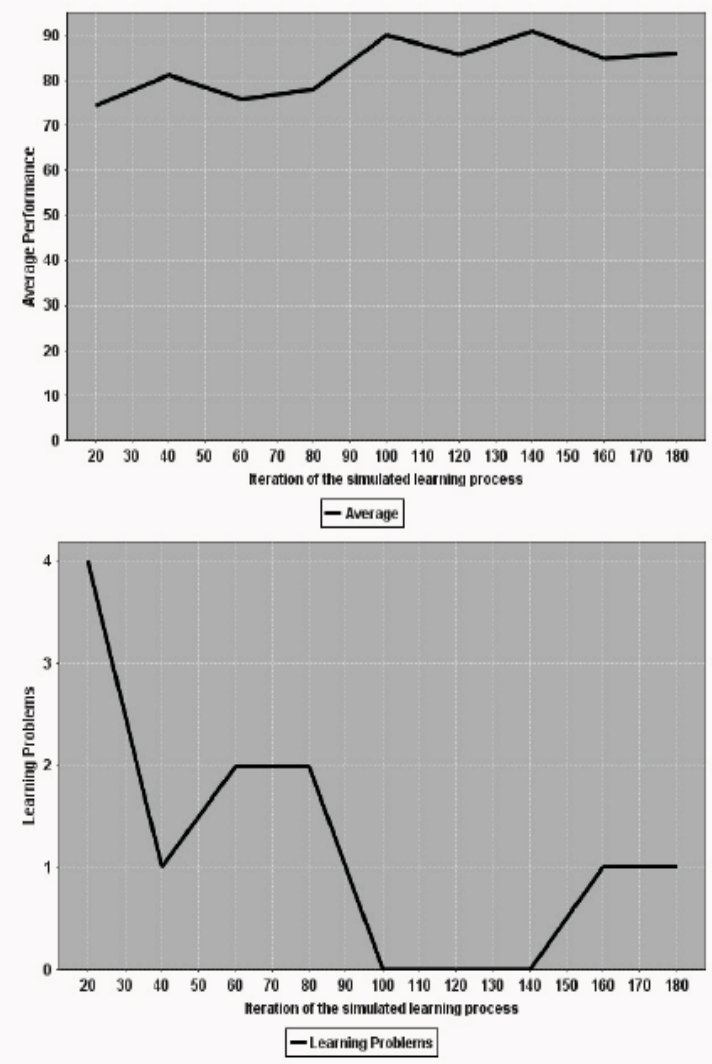

Fig. 5: Average performances and average learning problems in experiment 2

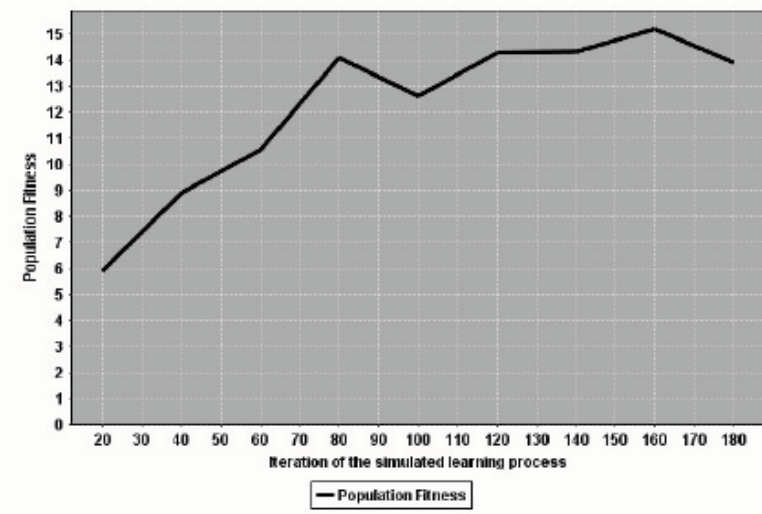

Fig. 6: Population Fitness during experiment 2

The LSr were the same as in Experiment 2. Figure 7 shows the results obtained from an execution of this experiment. As it can be seen, we had a considerable reduction of iterations, due to the initial consistency of LSp. Only two updates in LSp occurred during the learning process, which highlights the performance gain when SM

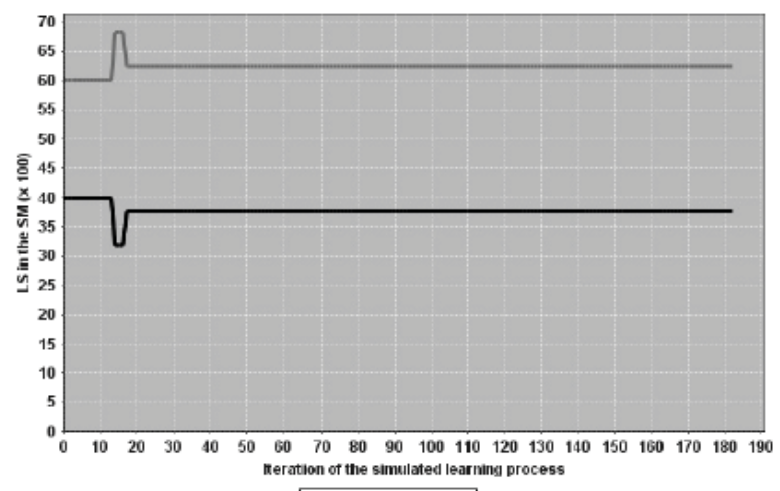

- Active - Reflective

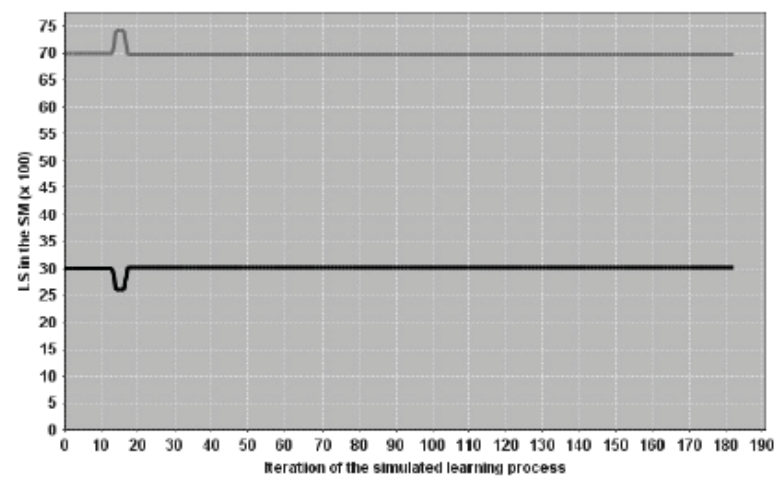

- Sensitive - Intuitive

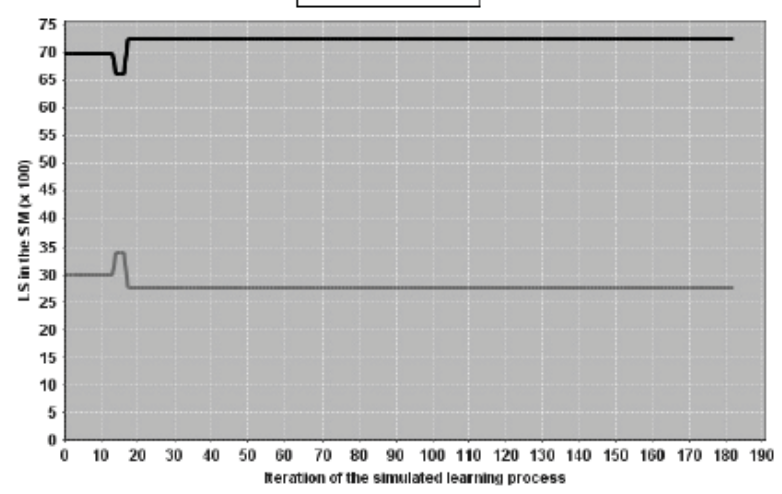

-Visual - Verbal

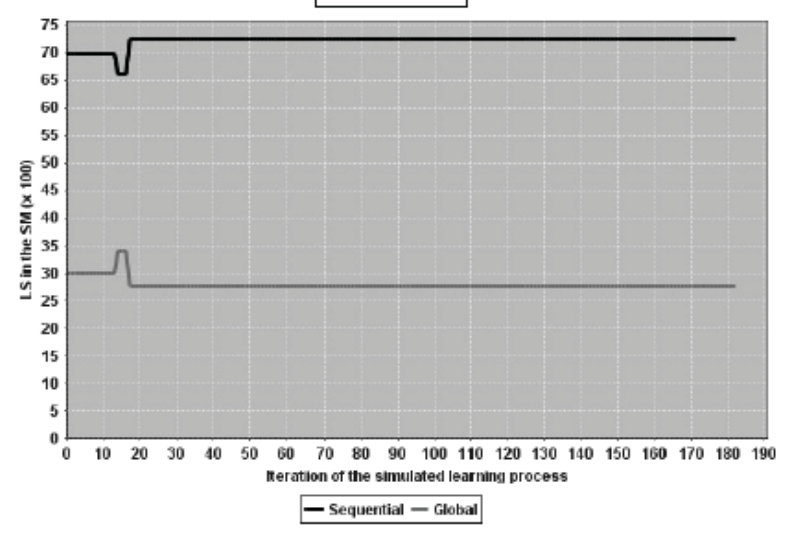

Fig. 7: Results from experiment 3 
is consistent and the learning process is well-fitted. Therefore, in this specific case, we had the occurrence of only two learning problems throughout the learning process, which is an ideal situation in a distance learning environment. As we know, this scenario is possible only when the system provides well-fitted content and feedback

to

the

student.
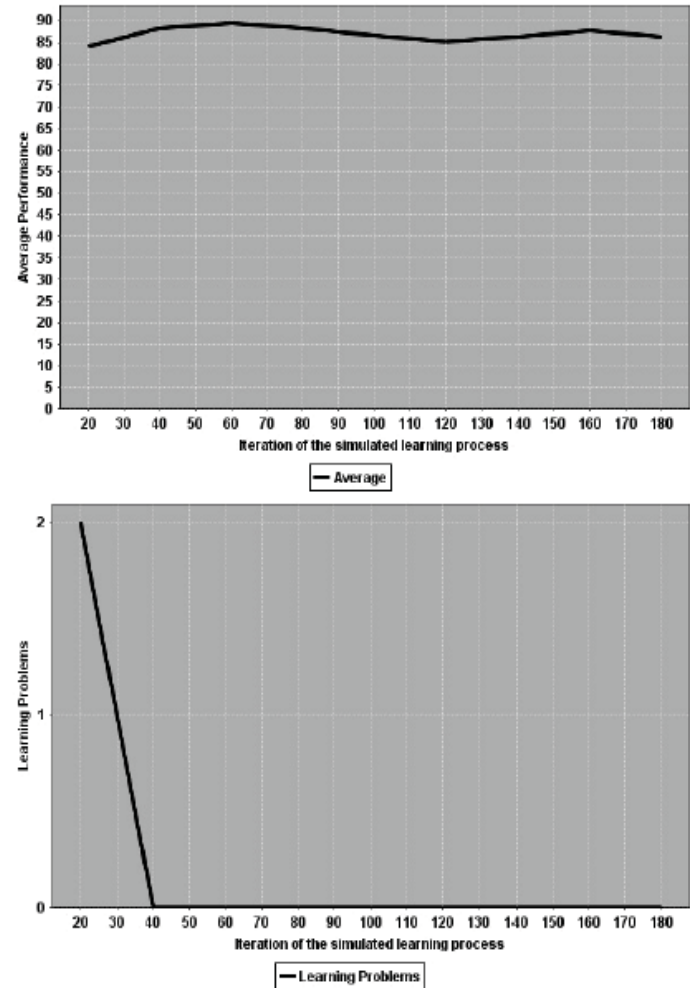

Fig. 8: Average performances and average learning problems from experiment 3

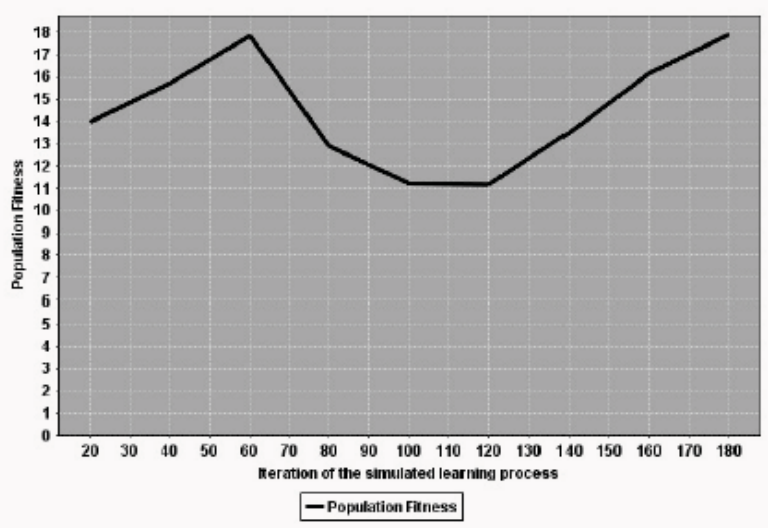

Fig. 9: Population Fitness in experiment 3
Figure 8 shows the average performance achieved by the student and the average learning problems in steps of 20 learning sessions. The average performance was initially high, and remained high throughout the learning process.

Figure 9 shows the population fitness. In this case, the population fitness was initially high, and it was increased throughout the learning process.

\subsection{Experiment 4}

This experiment considers the case in which students LSp were initially inconsistent in its four dimensions: LSp $=\{(0.80,0.20),(0.80,0.20),(0.80,0.20),(0.80,0.20)\} . \mathrm{LSr}$ $=\{$ reflective (weak), intuitive (strong), verbal (moderate), global (weak) $\}$. As it can be seen, in Figure 10, all of the student's preferences were efficiently detected. The learning process took 208 iterations, in which 28 learning problems occurred. The dimensions active/reflective, sensitive/intuitive and visual/verbal were efficiently corrected, becoming consistent in the very beginning of the learning process. However, the sequential/global dimension took more iterations to become consistent, and in the end of the learning process it became inconsistent again. This behaviour can be easily understood by observing that the student has a weak (or balanced) preference in this dimension. As pointed out by [13], balanced preferences are not well defined, and the student can do well in both, and interchange between the two, depending on the situation.

Figure 11 shows the average performance achieved by the student and the average learning problems in steps of 20 learning sessions. It is important to notice that the average performance was increased and the learning problems were decreased throughout the learning process. Figure 12 shows the population fitness.

The population fitness was increased as the SM became consistent. Finally, we believe that the results obtained from these experiments validate the proposed approach, which can be easily implemented in an existing LMS, like Moodle [32], and tested with real students. Simulating students performance based on their real LS and current SM was a very important part of our work, because it allowed us to test, adjust and correct our approach since the very beginning, optimizing the development process.

These adjustments and improvements were mainly related to the rules depicted in Section 3, which determine the updates in LSp, and to the calculation of the reinforcement applied by those rules. Both are crucial points of our work, which are still being adjusted. 

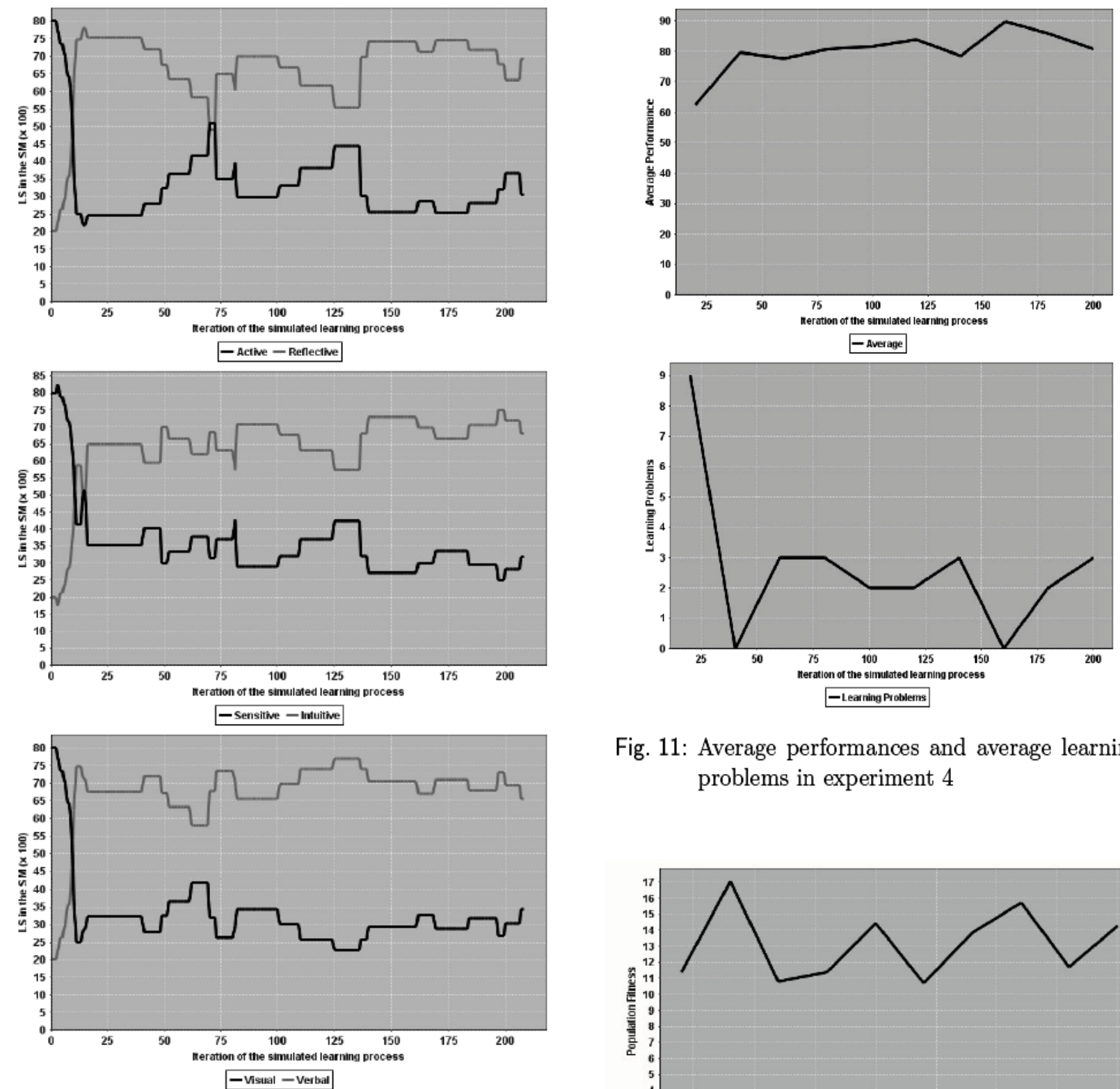

Fig. 11: Average performances and average learning problems in experiment 4

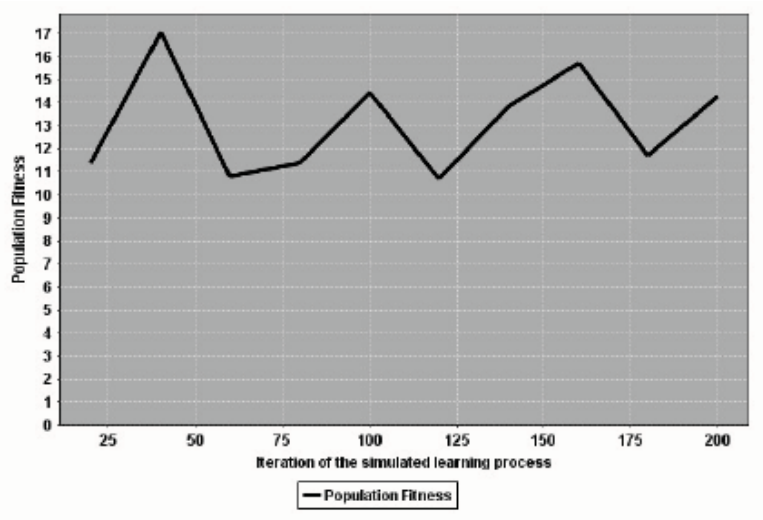

Fig. 12: Population Fitness in experiment 4

Therefore, without using simulation, it should be impossible to come up with this approach within reasonable time, due to the large amount of time necessary to do experiments with real students. A variety of experiments were performed - considering different real LS and initial LSp - and some of them have been exposed in this section. Next section points out some conclusions and future 
work.

\section{Conclusion and Future Works}

AES has been considered as a promising approach to increase the efficiency in computer-aided learning. A necessary characteristic in this approach is the precise, dynamic and continuous identification of students LS, in order to provide well-adapted learning experiences. In this context, one challenge is the development of systems able to efficiently acquire students LS.

The information about students LS acquired by psychometric instruments encloses some degree of uncertainty $[34,35]$. Furthermore, in most of the existing approaches, the assumptions about students LS, once acquired, are no longer updated. In this context, this work presents a new approach to automatically detect and precisely adjust a student's LS based on the nondeterministic and non-stationary aspects of LS, that may change during the learning process in an unexpected and unpredictable way [18].

Because of the probabilistic and dynamic factors enclosed on automatic detection of LS, our approach gradually and constantly modifies the SM through reinforcement learning, considering students performances as rewards of a pedagogical actions, represented by LSCs. In this way, LSCs are actions that the system can execute with the intention of teaching content, maximizing the quality of students performances in the learning process. Therefore, the reinforcement is calculated according to the performance value obtained by the student during a learning session. Consequently, SM converges towards the real students LS, as shown in section 4.

Using genetic algorithms techniques was crucial in order to handle the probabilistic aspect related to the selection of appropriate LSCs, considering that the students preferences are unknown. In order to validate the proposed approach, two variables were observed in the experiments: consistency and efficiency. Considering these variables, we could analyse if the SM were effectively corrected, and how fast it was corrected. We could notice that the proposed approach solves some important problems ignored by most of the analysed approaches, and brings advantages, due to specific points, as shown in section 2 .

In this way, an important aspect of the proposed approach is to consider that not only LS but many factors have an influence on students performances, making it harder to infer students LS based only on fixed behavioral pattern rules. Some of these factors are pointed out in [24, $22,28,2,23]$. The test and validation of our approach was initially done through computer simulation of students performance, which took into account some im- portant aspects on how LS influences students performances, as described by [24, 22, 28, 2, 23]. Evaluation of AES is a difficult task, as pointed out in [3]. Therefore, the validation of our model through simulation was vital, due to the time and human resources needed to test it using real students.

Now that we have achieved good results through simulation, we feel safe to use our approach in an existing LMS, like Moodle [32], and test it with real courses and students.

\section{References}

[1] S.C. Abdullah and R.E. Cooley. Using simulated students to evaluate an adaptive testing system. In Computers in Education, 2002. Proceedings. International Conference on, pages 614-618. IEEE, 2002. ISBN 0-7695-1509-6.

[2] E. Alfonseca, R.M. Carro, E. Martín, A. Ortigosa, and P. Paredes. The impact of learning styles on student grouping for collaborative learning: a case study. User Modeling and User- Adapted Interaction, 16(3):377401, 2006. ISSN 0924-1868.

[3] J. Bravo and A. Ortigosa. Validating the evaluation of adaptive systems by user profile simulation. In Proceedings of Workshop held at the Fourth International Conference on Adaptive Hypermedia and Adaptive Web-Based Systems (AH2006), pages 479-483, 2006.

[4] P. Brusilovsky. Adaptive educational hypermedia. In International PEG Conference, pages 8-12. Citeseer, jun 2001. [5] R.Z. Cabada, M.L.B. Estrada, and C.A.R Garcia. A Fuzzy-Neural Network for Classifying Learning Styles in a Web 2.0 and Mobile Learning Environment. In Web Congress, 2009. LEWEB' 09. Latin American, pages 177-182. IEEE, 2009.

[6] C. Carmona and G. Castillo. Designing a Dynamic Bayesian Network for Modeling Students Learning Styles. In Eighth IEEE International Conference on Advanced Learning Technologies, pages 346-350. IEEE, 2008.

[7] C. Carmona, G. Castillo, and E. Millán. Discovering Student Preferences in E-Learning. In Proceedings of the International Workshop on Applying Data Mining in e-Learning, 2007.

[8] G. Castillo, J. Gama, and A.M. Breda. An Adaptive Predictive Model for Student Modeling. Advances in Web-based education: Personalized learning environments, pages 70-92, 2005.

[9] W.C. Chang and M.S. Chung. Automatic applying 
Bloom's taxonomy to classify and analysis the cognition level of English question items. In Pervasive Computing (JCPC), 2009 Joint Conferences on, pages 727-734. IEEE, 2010.

[10] A. Chipperfield, P. Fleming, H. Pohlheim, and C. Fonseca. Genetic algorithm toolbox for use with matlab. Citeseer, 1994.

[11] F. Coffield, D. Moseley, E. Hall, and K. Ecclestone. Learning styles and pedagogy in post-16 learning: A systematic and critical review. National Centre for Vocational Education Research (NCVER), 2009. ISBN 1853389188.

[12] F. A. Dorça, C. R. Lopes, M. A. Fernandes, and R. S. Lopes. Adaptativity Supported by Neural Networks in Web-based Educational Systems. Journal of Education, Informatics and Cybernetics (JEIC), 1, 2009.

[13] R.M. Felder and L.K. Silverman. Learning and teaching styles in engineering education. Journal of Engineering education, 78(7):674-681, 1988.

[14] R.M. Felder and J. Spurlin. Applications, reliability and validity of the index of learning styles. International Journal of Engineering Education, 21(1):103112, 2005.

[15] A. L. Franzoni and S. Assar. Student learning styles adaptation method based on teaching strategies and electronic media. Educational Technology \& Society, 12(4):15-29, 2009.

[16] P. García, A. Amandi, S. Schiaffino, and M. Campo. Evaluating Bayesian networks' precision for detecting students' learning styles. Computers \& Education, 49(3):794-808, 2007. ISSN 0360-1315.

[17] D.E. Goldberg. Genetic algorithms in search, optimization, and machine learning. Addisonwesley, 1989. ISBN 0201157675.

[18] S. Graf and Kinshuk. Advanced Adaptivity in Learning Management Systems by Considering Learning Styles. In Proceedings of the 2009 IEEE/WIC/ACM International Joint Conference on Web Intelligence and Intelligent Agent Technology-Volume 03, pages 235-238. IEEE Computer Society, 2009.

[19] S. Graf and C.I. Kinshuk. A Flexible Mechanism for Providing Adaptivity Based on Learning Styles in Learning Management Systems. In $10^{\text {th }}$ IEEE International Conference on Advanced Learning Technologies, pages 30-34. IEEE, 2010.

[20] S. Graf and K. Kinshuk. Using Cognitive Traits for Improving the Detection of Learning Styles. In Database and Expert Systems Applications (DEXA), 2010 Workshop on, pages 74-78. IEEE, 2010.
[21] S. Graf and T.C. Liu. Identifying Learning Styles in Learning Management Systems by Using Indications from Students' Behaviour. In Advanced Learning Technologies, 2008. ICALT'08. Eighth IEEE International Conference on, pages 482-486. IEEE, 2008.

[22] S. Graf, Tzu-Chien Liu, and Kinshuk. Interactions Between Students Learning Styles, Achievement and Behaviour in Mismatched Courses. In Proceedings of the International Conference on Cognition and Exploratory Learning in Digital Age (CELDA 2008), pages 223-230. IADIS International Conference, 2008.

[23] S. Graf, C.H. Lan, T.C. Liu, et al. Investigations about the Effects and Effectiveness of Adaptivity for Students with Different Learning Styles. In 2009 Ninth IEEE International Conference on Advanced Learning Technologies, pages 415-419. IEEE, 2009.

[24] M.T.U. Haider, A.K. Sinha, and B.D. Chaudhary. An Investigation of relationship between learning styles and performance of learners. International Journal of Engineering Science and Technology, 2(7):28132819, 2010.

[25] IEEE. LOM (Learning Object Metadata). IEEE Learning Technology Standards Committee, http://ltsc.ieee.org/wg12/index.html, 2010.

[26] C. Jones, C. Reichard, and K. Mokhtari. Are Students Learning Styles Discipline Specific? Community College Journal of Research and Practice, 27(5):363-375, 2003. ISSN 1066-8926.

[27] D. Kelly and B. Tangney. 'First Aid for You': getting to know your learning style using machine learning. In Advanced Learning Technologies, 2005. ICALT 2005. Fifth IEEE International Conference on, pages 1-3. IEEE, 2005. ISBN 0769523382.

[28] Kinshuk, T.C. Liu, and S. Graf. Coping with Mismatched Courses: Students' behaviour and performance in courses mismatched to their learning styles. Educational Technology Research and Development, 57(6):739-752, 2009. ISSN 1042-1629.

[29] J.S. Mertz. Using a simulated student for instructional design. International Journal of Artificial Intelligence in Education (IJAIED), 8: 116-141, 1997.

[30] S. Messick. Personal styles and educational options. Individuality in learning, pages 327-368, 1976.

[31] S.P. Meyn, R.L. Tweedie, and P.W. Glynn. Markov chains and stochastic stability. Springer London, 1996. ISBN 3540198326.

[32] Moodle. http://www.moodle.org/, 2010.

[33] A. Papoulis, S.U. Pillai, and S. Unnikrishna. Proba- 
bility, random variables, and stochastic processes, volume 73660116. McGraw-Hill New York, 2002.

[34] L. Price. Individual differences in learning: Cognitive control, cognitive style, and learning style. Educational Psychology, 24(5):681-698, 2004. ISSN 01443410 .

[35] M.J. Roberts and G. Erdos. Strategy selection and metacognition. Educational Psychology, 13 (3):259266, 1993. ISSN 0144-3410.

[36] C.W. Starr, B. Manaris, and R.A.H. Stalvey. Bloom's taxonomy revisited: specifying assessable learning objectives in computer science. ACM SIGCSE Bulletin, 40(1):261-265, 2008. ISSN 00978418 .

[37] R.S. Sutton and A.G. Barto. Reinforcement learning: An introduction, volume 116. Cambridge Univ Press, 1998.

[38] K. Vanlehn, S. Ohlsson, and R. Nason. Applications of simulated students: An exploration. Journal of artificial intelligence in education, 5: 135-135, 1994. ISSN 1043-1020.

[39] M. Virvou, K. Manos, and G. Katsionis. An evaluation agent that simulates students' behaviour in intelligent tutoring systems. In IEEE International Conference on Systems, Man and Cybernetics, 2003, volume 5, pages 4872-4877. IEEE, IEEE, 2003. ISBN 0780379527 .

[40] A. Vizcaino and B. du Boulay. Using a simulated student to repair difficulties in collaborative learning. In ICCE '02 Proceedings of the International Conference on Computers in Education. ACM, IEEE Computer Society Washington, DC, USA, 2002.

[41] M. R. Zakaria and T. J. Brailsford. User Modeling and Adaptive Educational Hypermedia Frameworks for Education. New Review of Hypermedia and Multimedia, 1(8):83-97, 2002.

[42] R. Zatarain, L. Barrón-Estrada, C. Reyes-García, and O. Reyes-Galaviz. Applying Intelligent Systems for Modeling Students' Learning Styles Used for Mobile and Web-Based Systems. Soft Computing for Intelligent Control and MobileRobotics, pages 3-22, 2010.

[43] R. Zatarain-Cabada, M. Barrón-Estrada, L. ZepedaSánchez, G. Sandoval, J. Osorio-Velazquez, and J. Urias-Barrientos. A Kohonen Network for Modeling Students' Learning Styles in Web 2.0 Collaborative Learning Systems. MICAI 2009: Advances in Artificial Intelligence, pages 512-520, 2009. 